\title{
DNS: A STATISTICAL ANALYSIS OF NAME SERVER TRAFFIC AT LOCAL NETWORK-TO- INTERNET CONNECTIONS
}

\author{
Chris Brandhorst ${ }^{1}$ and Aiko Pras ${ }^{2}$ \\ ${ }^{\prime}$ Faculty of Electrical Engineering, Mathematics and Computer Science, University of Twente, \\ Enschede, $7522 \mathrm{NK}$, The Netherlands \\ c.j.brandhorst@cs.utwente.nl \\ ${ }^{2}$ Faculty of Electrical Engineering, Mathematics and Computer Science, University of Twente, \\ Enschede, P.O. BOX 217, 7500 AE Enschede, The Netherlands \\ pras@cs.utwente.nl
}

\begin{abstract}
This paper puts forward a purely statistical analysis of name server traffic captured at four different locations: two links to residential networks, and two to the Dutch academic and research institute. Analysis of the system can give insight in the use and performance of the protocol, which is helpful for future improvement. Multiple analyses can show the development of the performance over time and help create quality models. The analysis shows that a little more than $12 \%$ of all queries are not answered upon. Three quarters of the lookups are successful: they give the client the correct IP address mapping for the requested hostname. $90 \%$ is answered within $275 \mathrm{~ms}$, with an average of $152 \mathrm{~ms}$. In $9 \%$ of all cases, clients ask for a hostname which does not exist. At one of the locations, a client is discovered which sends queries to two DNS servers at a remarkable rate: one each 11 to $22 \mathrm{~ms}$.
\end{abstract}

Keywords: DNS, Internet, measurement, performance

\section{INTRODUCTION}

The Domain Name System is one of the most vital protocols of the modern Internet. This protocol is responsible for translating Internet addresses (e.g. www.server.com) to network locations; also called IP 
addresses (e.g. 125.114.163.15). This translation occurs at DNS servers to which a client must send its queries, asking for the IP address of a specific Internet address. The DNS is vital, because virtually all applications that connect to the Internet (e.g. mail programs, web browsers and FTP utilities) make use of the protocol for their operation. Therefore, it is necessary that the system must be operable virtually $100 \%$ and have a high degree of efficiency, to provide the clients with low response times to there queries.

Analysis of the DNS traffic will give insight in the use of the protocol and may assist in improving it in the future or designing a new version. Multiple analyses can show the development of the use of the protocol over time and "studies involving certain DNS performance measures would be greatly strengthened by data from many locations"[Liston et al., 2002]. This, in turn, can address performance issues. Furthermore, when these statistics are known, quality models can be created for evaluating new protocols, algorithms and architectures [ACM, 2004].

\subsection{Related Work}

Past work is available on this subject. The most quoted of them was done by [Danzig et al., 1992]. Others are [Brownlee et al., 2001], [Jung et al., 2002] and [Liston et al., 2002]. This work will differ from the above in three aspects.

The first is that it will take measurements from at least four local network-to-Internet connections, all with a varying number of users, types of users and bandwidth. Jung et al. use data gathered at just two networks, both of which belong to research institutes and Liston et al. gather their data at 75 client locations, which is far less than the number of clients connected to the networks used in this study.

The second difference is the topographical network location at which the measurements have taken place. The data used for this research was captured at the switches which connect the local networks to the Internet. Danzig et al. and Brownlee et al. have concentrated on measurements at a DNS root server, where the first also takes three domain servers into account. Liston et al. have taken a different approach by measuring the DNS performance at client computers. One can say that this research can be placed closer to Liston's, because of the proximity of the clients to the switch.

Thirdly, only Jung et al. include (limited) basic trace statistics in their research. In this research an extensive breakdown of all DNS responses will be given. 


\subsection{Research Questions}

There are a number of statistics that can be extracted from DNS traffic data. This analysis will include a breakdown of DNS queries into those that: are erroneous, are refused, ask for non-existing domains, are omitted because of a server failure, are not replied to at all, are of a type which is not implemented by the receiving DNS server and those that are answered normally. Besides this, information can be gathered about the delay between the sending and receiving of DNS queries, also called latency, how many queries are to be processed recursively and which fraction of the total traffic can be accounted for by the DNS.

This results in the following research questions, by which the performance of the DNS, as perceived by the client, can be analyzed:

1. What is the fraction of total traffic that can be accounted for by the DNS, measured in packets as well as bytes?

2. What fraction of the received DNS queries is to be processed recursively by the DNS servers?

3. Given a collection of DNS queries, which fractions of these queries are answered normally and which fraction results in some kind of error? We will determine the fractions of the following error conditions: queries that 1) are erroneous, 2) are refused by the DNS servers, 3) ask for nonexisting domains, 4) are omitted because of a server failure, 5) are not replied to at all, and 6) are of a type which is not implemented by the receiving DNS servers.

4. What is the average delay between the time that the query is sent and the answer is received? (the measured delay is the time measured at the place in the network where the DNS data is collected, not the latency the client observes)

5. What are the differences between these results and prior research on DNS traffic, mostly focused on that of [Jung et al., 2002]?

In order to answer the above research questions, the repository set up by the M2C-project (Measuring, Modeling and Cost Allocation) at the University of Twente will be used [M2C]. This collection includes data captured at four locations, at different times. The locations include Ethernet links which connect 1) a residential network of a university to the core network of this university, 2) a research institute to the Dutch academic and research network, 3) a large college to that same research network and 4) a couple of hundred ADSL customers, mostly student dorms to an aggregated uplink of an ADSL access network.

In the following chapter a general overview of the Domain Name System is given. After this, the method of research is laid out in chapter three and in 
chapter four the results will be presented out of which conclusions will be drawn in the final chapter.

\section{THE DOMAIN NAME SYSTEM}

The Domain Name System is a standard and is defined in RFCs 1034 [Mockapetris, 2004] and 1035 [Mockapetris, 2004]. Here, only an overview of the most important basic functionality is given.

The Internet's DNS function is to translate human-readable hostnames (e.g. www.server.com) to IP addresses (e.g. 125.114.163.15), which are used by network-hardware to identify client computers. This translation can be done for all kinds of servers, from web- and mail- to FTP-servers and is employed by the corresponding application-layer protocols (HTTP, POP/SMTP and FTP respectively), which are in turn used by computer applications. The application will ask the DNS for the IP address of the host of which the client entered the hostname by sending a DNS query onto the network. After a delay, the client will receive a response stating the hostname or possibly an error message.

The DNS can be used on top of both the UDP and TCP protocol.

\subsection{Name Servers}

To achieve its functionality, the DNS consists of a large number of name servers which are scattered around the world and ordered hierarchical. There is not one server that has DNS records for all hostname to IP-mappings for the Internet. Instead, a certain DNS server has records for a specific domain (like .nl or utwente.nl) or local network. Besides this, it can route DNS queries for mappings that that server does not contain to a DNS server which does. Therefore, there are two kinds of name servers [Jung et al., 2002]:

- Root name servers. If the name server in the local network of the client does not have a mapping for the requested hostname, the local name server sends a query to one of the 13 root name servers [root-servers, 2004]. These servers contain DNS records of authoritative name servers, one of which is returned to the local name server.

- Authoritative name servers. A name server is authoritative for a certain host or domain if it contains DNS records for that host or domain or has a record for another DNS server that does has these records. This layering of DNS servers can be repeated more than once. 


\subsection{Recursive Queries}

An example of a recursive query is shown in Figure 1. The query and its response together often are called a lookup. The host 192.168.1.203 here requests the IP address of www.utwente.nl. It sends this query to its local name server, 192.168.1.1. The local server does not have the correct mapping, so it sends a query to the root server (b.root-servers.net). This root server knows the address of the authoritative name server for the.nI top-level-domain (ns.domain-registry.n1), which is returned to the local name server. This server in turn, when asked by the local name server, returns the address of the authoritative name server for the utwente.nl. domain (ns1.utwente.nl). Finally, after querying this server, the IP-address of www. utwente.n工 is obtained and returned to the client. This query is called recursive, because the client only sends one query and receives one answer. The local name server handles all the other queries in favor of the client. When a query is iterative, it means that the name server to which the query is sent will always send a response to the requesting host instead of to an other name server, whether it does have a DNS record for the requested hostname (it will send the requested IP address) or it does not (it will send the IP address of an authoritative name server). Not all name servers support recursive querying. Note that the local name server in the example only sends iterative queries.

\subsection{Response Types}

When a query is answered by a name server, this response is of one of the following types [Mockapetris, 2004]:

- OK. Everything went correctly and embedded in this response packet is the IP address belonging to the hostname the requesting host supplied.

- Format error. The name server was unable to interpret the query.

- Server failure. The name server was unable to process this query due to a problem with the name server.

- No such name. The domain name referenced in the query does not exist.

- Not implemented. The name server does not support the requested kind of query.

- Refused. The name server refuses to perform the specified operation for policy reasons. For example, a name server may not wish to provide the information to the particular requester, or a name server may not wish to perform a particular operation for particular data. 


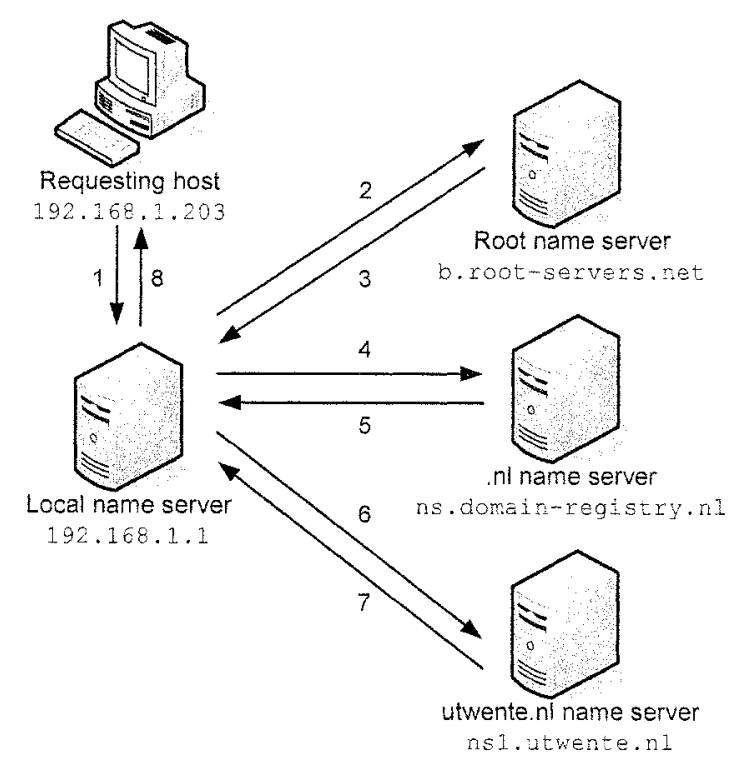

Figure 1. An example of a recursive query [Wanrooij, 2005].

\section{$2.4 \quad$ Latency}

The time between the sending of a DNS query and the receiving of a response is called the latency of that query. The latency can vary between milliseconds and tens of seconds [Kurose and Ross, 2003].

\section{RESEARCH METHODOLOGY}

\subsection{Used Data}

In order to analyze the DNS traffic, this study makes use of the Internet data repository set up by the $\mathrm{M} 2 \mathrm{C}$ project (Measuring, Modeling and Cost Allocation) [M2C, 2004]. This repository consists of 555 traces (in December 2004), each of which contains 15 minutes of packet data. The measurements were taken at four different locations, all in the Netherlands, on different days and times [M2C, 2004].

"On location \#1 the $300 \mathrm{Mbit} / \mathrm{s}$ (a trunk of $3 \times 100 \mathrm{Mbit} / \mathrm{s}$ ) Ethernet link has been measured, which connects a residential network of a university to the core network of this university. On the residential network, about 2000 students are connected, each having a $100 \mathrm{Mbit} / \mathrm{s}$ Ethernet access link. The residential network itself consists of 100 and $300 \mathrm{Mbit} / \mathrm{s}$ links to the various 
switches, depending on the aggregation level. The measured link has an average load of about $60 \%$. Measurements have taken place in July 2002" and are spread over 15 traces.

1. "On location \#2, the $1 \mathrm{Gbit} / \mathrm{s}$ Ethernet link connecting a research institute to the Dutch academic and research network has been measured. There are about 200 researchers and support staff working at this institute. They all have a $100 \mathrm{Mbit} / \mathrm{s}$ access link, and the core network of the institute consists of $1 \mathrm{Gbit} / \mathrm{s}$ links. The measured link is only mildly loaded, usually around $1 \%$. The measurements are from May to August 2003" and are spread over 183 traces.

2. "Location \#3 is a large college. Their $1 \mathrm{Gbit} / \mathrm{s}$ link (i.e., the link that has been measured) to the Dutch academic and research network carries traffic for over 1000 students and staff concurrently, during busy hours. The access link speed on this network is, in general, $100 \mathrm{Mbit} / \mathrm{s}$. The average load on the $1 \mathrm{Gbit} / \mathrm{s}$ link usually is around $10-15 \%$. These measurements have been done from September - December 2003" and are spread over 255 traces.

3. "On location \#4, the $1 \mathrm{Gbit} / \mathrm{s}$ aggregated uplink of an ADSL access network has been monitored. A couple of hundred ADSL customers, mostly student dorms, are connected to this access network. Access link speeds vary from $256 \mathrm{kbit} / \mathrm{s}$ (down and up) to $8 \mathrm{Mbit} / \mathrm{s}$ (down) and 1 Mbit/s (up). The average load on the aggregated uplink is around 150 Mbit/s. These measurements are from February - July 2004" and are spread over 102 traces.

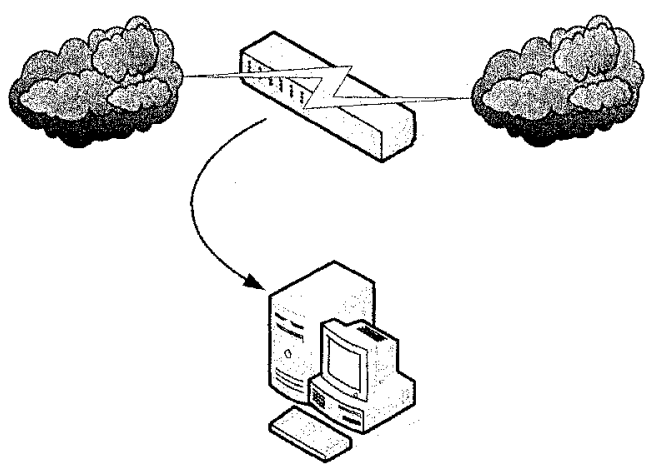

Figure 2. The setup for the collection of the packet data [Van de Meent, 2003]. 


\subsection{Method of Collection}

For every location, the method of collection was identical. "The measurements are performed by capturing the headers of all packets that are transmitted over the (Ethernet) 'uplink' of an access network to the Internet." [Van de Meent, 2003], see also Figure 2. To the switch (can also be a router), a measurement PC was connected. To capture the 15 minutes of packets into binary files, the utility tcpdump [TCPDUMP, 2004] was used. These traces were anonymized, compressed and published on the Internet [M2C, 2004][Van de Meent, 2003].

The anonymization is done for privacy reasons, which means 1) that only the packet headers are captured, so it is unknown what data is sent from the network to the Internet and 2) that the source and destination IP addresses of the packets are scrambled consequently, so that is unknown from and to which host the packets are sent. But within a trace, a single IP address is each time scrambled to the same anonymized IP address and two IP addresses that are within the same network, will have anonymized counterparts that also can be placed within the same network. For a more detailed explanation of this method, see [Van de Meent, 2003].

In section II it was said that the DNS protocol can be used on top of both TCP and UDP, but a few samples from each location revealed zero DNS packets that made use of the TCP protocol. Therefore it is assumed in this paper that the use of this protocol for the Internet is negligible.

\subsection{Method of Analysis}

For the analysis of the data, a self-built Java application called ReadDump was developed. This application opens a packet-trace file and analyses the packets within to retrieve the data necessary to answer the above research questions. When it is done, it writes a report to a log file. Because there are 555 traces, another program was developed to send the data files to the Java so it could analyze all data autonomously. Runner was written in VB.NET and for each packet trace file, it would decompress it and send it to ReadDump for processing.

\section{RESULTS}

In Table 1 and 2, the statistical results of the analysis are presented. 


\subsection{Location \#1: Server Failures}

What immediately came to attention is the enormous amount of returned server failures at location \#1 (see Table 1). $60.99 \%$ of all sent queries get this response. The first thought was that a broken DNS server was the cause of these high amounts of server failures. After further research [Van de Meent, 2004], it seemed that not a DNS server but (most probably) one single client was the cause. This will be discussed further in section 4.1. Because this abnormal behavior was observed in 7 of the 15 traces at location \#1, only the remaining 8 traces were used to calculate new statistics which are laid out in Table 2. Important to note here is that retransmissions of DNS queries are taken into account as a 'new' query. The retransmissions cannot be measured because of the anonymized nature of the repository; it cannot be shown when a query is retransmitted, because of the lack of the real data of the packets.

Table 1. Trace statistics*

\begin{tabular}{|c|c|c|c|c|}
\hline & Location $\# 1$ & & All & \\
\hline Date & $2002 / 05 / 23-06 / 26$ & & & \\
\hline Total Gigabytes & 263.3 & & $2,487.5$ & \\
\hline DNS Megabytes (1) & 139.8 & $(0.05 \%)$ & $1,220.3$ & $(0.05 \%)$ \\
\hline Total packets & $429,846,054$ & & $4,085,445,051$ & \\
\hline DNS packets (2) & $1,556,032$ & $(0.36 \%)$ & $10,450,329$ & $(0.26 \%)$ \\
\hline Queries (3) & 780,538 & $(50.16 \%)$ & $5,527,878$ & $(52.90 \%)$ \\
\hline Recursive (4) & 670,425 & $(85.89 \%)$ & $1,011,762$ & $(18.30 \%)$ \\
\hline Iterative (4) & 110,113 & $(14.11 \%)$ & $4,516,116$ & $(81.70 \%)$ \\
\hline Unanswered (4) & 5,044 & $(0.65 \%)$ & 605,427 & $(10.95 \%)$ \\
\hline OK $(4)$ & 233,965 & $(29.97 \%)$ & $3,715,741$ & $(67.22 \%)$ \\
\hline Format error (4) & 667 & $(0.09 \%)$ & 167,726 & $(3.03 \%)$ \\
\hline Server failure (4) & 476,055 & $(60.99 \%)$ & 536,729 & $(9.71 \%)$ \\
\hline No such name (4) & 64,171 & $(8.22 \%)$ & 450,601 & $(8.15 \%)$ \\
\hline Not implemented (4) & 274 & $(0.04 \%)$ & 4,145 & $(0.07 \%)$ \\
\hline Refused (4) & 362 & $(0.05 \%)$ & 47,509 & $(0.86 \%)$ \\
\hline Average latency (ms) & \multicolumn{2}{|c|}{$687, \sigma=364$} & \multicolumn{2}{|c|}{$156, \sigma=206$} \\
\hline
\end{tabular}

* Trace statistics including 7 traces in which a client with abnormal behavior is detected. Percentages are with respect to: (1) Total bytes; (2) Total \# of packets; (3) \# of DNS packets; (4) \# of Queries. 


\begin{tabular}{|c|c|c|c|c|c|c|c|c|c|c|c|c|c|c|c|c|c|c|}
\hline & $\overline{<}$ & & $\mid \begin{array}{c}a \\
0 \\
m \\
m \\
c\end{array}$ & 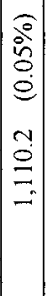 & $\mid \begin{array}{c}5 \\
\vdots \\
m \\
\sigma \\
\vdots \\
n \\
\infty \\
m \\
m\end{array}$ & 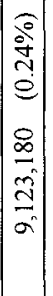 & 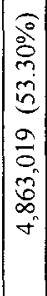 & 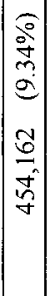 & 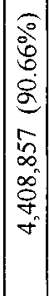 & 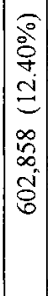 & 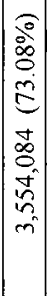 & $\mid \begin{array}{c}0 \\
\frac{0}{5} \\
0 \\
0 \\
8 \\
0 \\
0 \\
0 \\
0\end{array}$ & 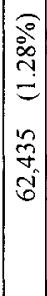 & 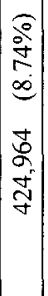 & 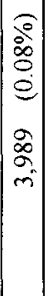 & $\mid \begin{array}{c}\hat{2} \\
\vdots \\
2 \\
e \\
9 \\
\hat{e} \\
\hat{2} \\
\tilde{y}\end{array}$ & $\left|\begin{array}{c}1 \\
0 \\
11 \\
0 \\
6 \\
0 \\
2\end{array}\right|$ & 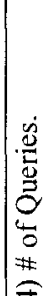 \\
\hline & 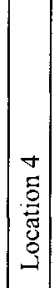 & 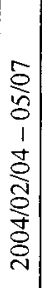 & $\mid \begin{array}{l}\tilde{0} \\
0 \\
\tilde{n} \\
-1\end{array}$ & 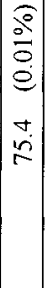 & $\mid \begin{array}{c} \\
\infty \\
\tilde{m} \\
\overrightarrow{2} \\
\overrightarrow{2} \\
\frac{\overrightarrow{0}}{\vec{v}} \\
\overrightarrow{\vec{v}}\end{array}$ & 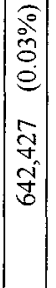 & 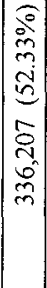 & 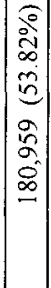 & 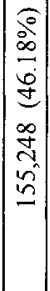 & $\mid \begin{array}{c}\widehat{0} \\
\hat{\alpha} \\
\alpha \\
\infty \\
\infty \\
\infty \\
\alpha \\
\hat{\jmath}\end{array}$ & 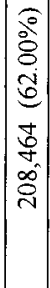 & $\begin{array}{c}0 \\
0 \\
0 \\
0 \\
0 \\
2 \\
2 \\
0 \\
\infty \\
-\end{array}$ & 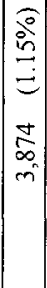 & 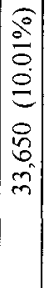 & 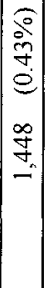 & $\begin{array}{c}0 \\
8 \\
\delta \\
\varrho \\
\Xi \\
8 \\
8 \\
8 \\
8 \\
8\end{array}$ & $\begin{array}{c}f \\
f \\
i 1 \\
0 \\
0 \\
n \\
m \\
m\end{array}$ & 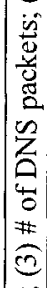 \\
\hline & 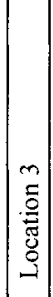 & 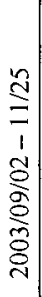 & $\left|\begin{array}{l}0 \\
1 \\
\infty \\
\infty\end{array}\right|$ & 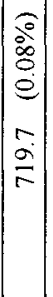 & 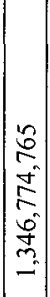 & 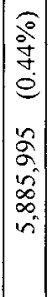 & 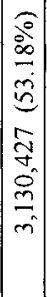 & 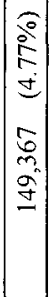 & 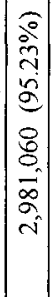 & 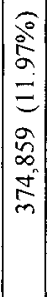 & $\mid \begin{array}{c}0 \\
0 \\
0 \\
0 \\
5 \\
5 \\
0 \\
0 \\
0 \\
0 \\
0 \\
0 \\
0\end{array}$ & 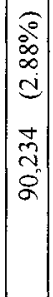 & 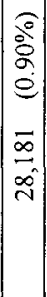 & 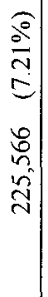 & 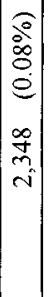 & 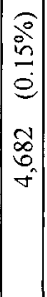 & $\mid \begin{array}{c}\infty \\
\infty \\
11 \\
0 \\
0 \\
\infty \\
\infty\end{array}$ & 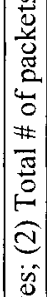 \\
\hline & 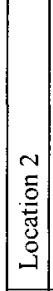 & $\begin{array}{l}\infty \\
0 \\
\infty \\
0 \\
1 \\
0 \\
0 \\
5 \\
0 \\
8 \\
8 \\
0\end{array}$ & $\frac{9}{5}$ & 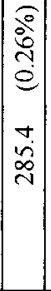 & $\mid$ & $\mid \begin{array}{c}0 \\
0 \\
7 \\
= \\
0 \\
0 \\
0 \\
0 \\
0 \\
0 \\
0 \\
0\end{array}$ & 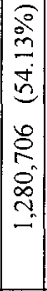 & $\mid \begin{array}{c}0 \\
8 \\
0 \\
0 \\
0 \\
\overline{0} \\
0 \\
= \\
=\end{array}$ & 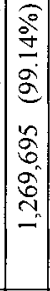 & 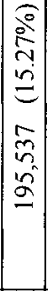 & 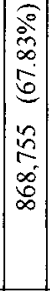 & 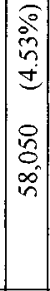 & 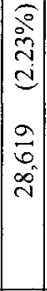 & 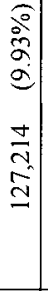 & $\mid \begin{array}{c}0 \\
0 \\
0 \\
0 \\
2\end{array}$ & 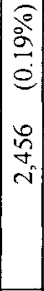 & $\left|\begin{array}{c}0 \\
0 \\
11 \\
0 \\
\pm \\
=\end{array}\right|$ & $\begin{array}{l}0 \\
\tilde{\pi} \\
\stackrel{0}{0} \\
\hat{\theta}\end{array}$ \\
\hline 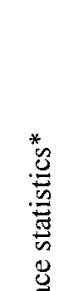 & 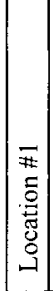 & 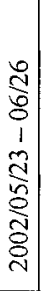 & $\begin{array}{l}2 \\
0 \\
0\end{array}$ & 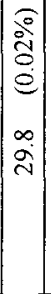 & 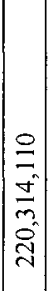 & 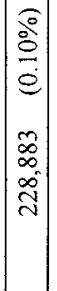 & $\begin{array}{c}0 \\
0 \\
0 \\
0 \\
0 \\
0 \\
0 \\
6 \\
6 \\
0 \\
=\end{array}$ & 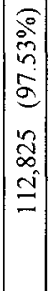 & 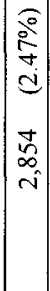 & 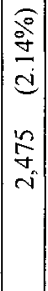 & 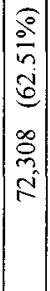 & 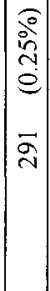 & 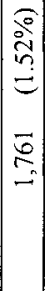 & 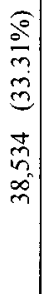 & $\begin{array}{c}\frac{\sigma}{0} \\
\stackrel{0}{e} \\
\infty \\
\equiv\end{array}$ & $\mid \begin{array}{l}2 \\
2 \\
2 \\
\alpha \\
\sigma\end{array}$ & $\left|\begin{array}{c}2 \\
2 \\
11 \\
0 \\
2 \\
\sigma\end{array}\right|$ & $\begin{array}{l}3 \\
u \\
u \\
z \\
z \\
0\end{array}$ \\
\hline 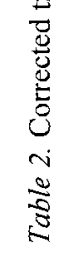 & & $=$ & 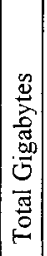 & 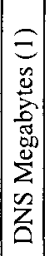 & 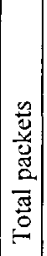 & 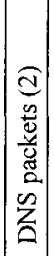 & 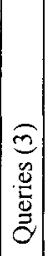 & 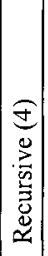 & 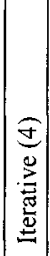 & 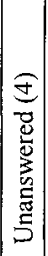 & $\begin{array}{c}\vec{J} \\
\frac{y}{0}\end{array}$ & 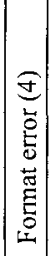 & 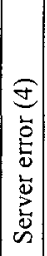 & 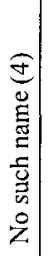 & 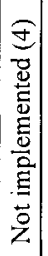 & 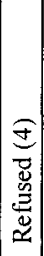 & 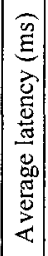 & 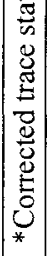 \\
\hline
\end{tabular}




\subsection{Statistics}

Several years have passed between the measurements which are used for this research and the results of the mentioned previous work, therefore adaptations to the DNS protocol may have been made too improve the performance. From the existing DNS RFCs, however, it followed that no adaptations were made in these years. The results can thus be properly compared.

\subsubsection{DNS Fraction of Total Traffic}

The percentage of total bytes which are DNS traffic varies little; between $0.01 \%$ and $0.26 \%$, on average $0.05 \%$. A little more variation is observed for the percentage of total packets which are DNS packets; between $0.03 \%$ and $1.41 \%$, on average $0.23 \%$. The data shows that there are two locations which in both cases have (by far) the least percentage of DNS traffic: locations \#1 and \#4.

\subsubsection{Recursive or Iterative}

Whether the queries are sent recursive, varies a great deal; between $0.86 \%$ and $97.53 \%$. Locations $\# 1$ and \#4 have high values for recursive queries, \#2 and \#3 show many iterative queries. This difference most probably comes from the fact that at locations \#2 and \#3, a DNS server that can receive recursive queries is present inside the local network. It can be assumed that the clients of those networks use those recursive DNS servers as a standard. This implies that virtually all DNS queries that are directed outside the local networks (the queries that are observed) will be iterative, because that traffic travels from the local DNS servers to a root server (see section II.B).

In contrast, at locations $\# 1$ and $\# 4$, the local DNS servers are placed outside the network. If we assume the same as for locations \#2 and \#3, this implies that all queries will be directed out of the network and measured, and because of the standard setup of the clients, these queries will be recursive. Still, at location \#4, the amount of recursive and iterative queries is almost equal. The reason for this may be a privately setup DNS server inside the network.

Jung reports similar variation in the number of recursive or iterative queries in the observed networks [Jung et al., 2002]. 


\subsubsection{Answering rates}

All locations show that a little more than $50 \%$ of all DNS packets consist of queries, implying that there are few queries that are not answered. Location \#2 tops with $15.27 \%$ of all queries not being answered, location \#1 has the least; $2.14 \%$. On average this is $12.40 \%$. Compared to the results of Jung et al., these DNSs perform far better; Jung reported 20.1-23.5\% unanswered [Jung et al. 2002].

\subsubsection{Return types}

Of the queries that were answered, on average $73.08 \%$ were queries for hostname mappings that could be correctly retrieved by the DNS server(s). Jung et al. found similar values (twice 64\% for two locations) and one far worse (36\% for the third location). As can be classified as logical, the percentage of no such name responses is not negligible. On average $8.74 \%$ of all requests are for hostnames that doesn't exist. Location \#1 tops here with $33.31 \%$. Jung also reports a large variation $(10 \% \text { to } 42 \%)^{5}$. Their explanation lies in "inverse lookups for IP addresses with no inverse mappings" and "particular invalid names such as loopback, and [...] records that point to names that do not exist." Because of the anonymized nature of the traces, it cannot be confirmed that the same causes for these rates are applicable here.

Not implemented and refused are returned rarely, with an exception at location $\# 4$, which has a high refused rate of $11.90 \%$. The reason for this cannot be uncovered. Format error is returned a little more, with tops of $4.53 \%$ and $5.58 \%$. The amount of server failure responses also is quite low.

\subsubsection{Latency}

The observed latency times are mostly low; on average $152 \mathrm{~ms}$. Location \#1 tops with 919 ms. Compared to some prior studies, this is far better. Wills and Shang [as quoted [Liston, 2002] ] "report lookup times exceeding 2.0 seconds for as many as $29 \%$ of lookups to random servers, and Cohen and Kaplan report lookup times exceeding 3.0 seconds for as many as $10 \%$ of lookups." Jung reports that $90 \%$ of all lookups on the MIT network had a latency of $447 \mathrm{~ms}$ in January 2000 and 11 months later, this number was about 2.5 times greater [Jung et al. 2002]. In our study merely 7 traces had an average latency of over 1 second and only one over than 2 seconds. Also, $90 \%$ of the traces have an average latency of $275 \mathrm{~ms}$ or less. $32 \%$ of all traces time between 75 and $100 \mathrm{~ms}$. See Figure 3 . 
It can be seen that at locations \#1 and \#4, the average latency is far greater compared to the other locations. If the assumptions made in 4.2.2 are correct, this difference can be addressed to the fact that the fraction of observed DNS recursive queries at locations \#1 and \#4 is far greater. A client that sends a recursive query gets a response only after all iterative queries that are needed to resolve the client's query are resolved by the recursive DNS server. So the total latency for a recursive query is higher than for an iterative query. This high latency can be seen at location \#1 and (to a lesser degree, because of the higher fraction of iterative queries) at location \#4, where the recursive queries from the clients to the DNS servers are observed. This in contrast to locations $\# 2$ and \#3, where the observed queries are (virtually all) the iterative queries sent from the local DNS servers to the root servers.

The higher standard deviation of the latency at locations \#1 and \#4 can be explained by noting that when sending a recursive query, a number of iterative queries are performed by the receiving DNS server. In the case of an iterative query, only one query is performed. Thus, the more iterative queries are performed which all have a deviation from the average, the higher the total standard deviation will be.

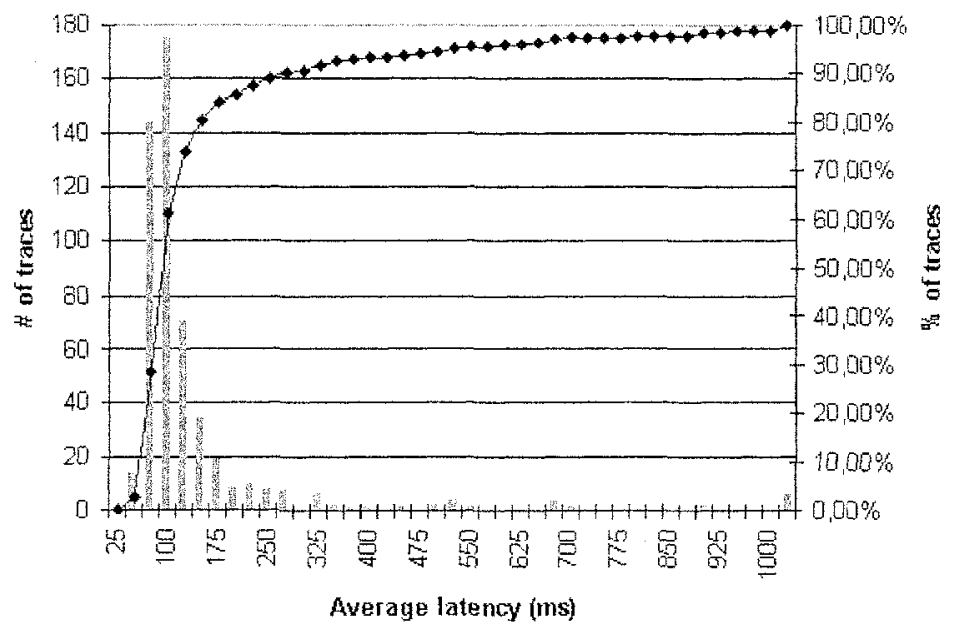

Figure 3. Cumulative latency distribution. 


\subsection{Client with abnormal DNS behavior}

It seemed that virtually $100 \%$ of the server failure responses within the 7 discarded location \#1 packets (see section IV.A) were directed to one single client. This cannot be said with full certainty, because in every trace the IP addresses were scrambled differently, but it is very reasonable to assume this.

This client was in each trace receiving DNS server failures from two different name servers. This receiving furthermore occurred at an exceptionally high rate: one received packet per 22 to $11 \mathrm{~ms}$. This rate of reception implies that the client should also have approximately the same rate of sending DNS queries.

So all 'evidence' points to a client that was either deliberately sending all these queries to the name server for some reason, or who's computer was misconfigured, which caused the continuing sending of queries. It was revealed that the name servers on the university network are specifically configured to answer queries for certain zones only when these queries come from specific clients [Van de Meent, 2004]. If any other client asks for an IP from one of these zones, a server failure is returned. This complies to a certain extent with RFC 2308 [Andrews, 2004], which declares that server failures fall in two classes, one of which states that "This may be where it (the name server, red.) has been listed as a server, but not configured to be a server for the zone, or where it has been configured to be a server for the zone, but cannot obtain the zone data for some reason." This 'some reason', most probably, is the fact that the DNS servers may not return the IP mappings for certain zones to any clients but the specified ones.

\section{CONCLUSION}

This paper put forward a statistical analysis of DNS data captured at four different locations: two links to residential networks, and two to the Dutch academic and research institute. The performance of the DNS, as seen by the client, was analyzed by measuring the latency and a breakdown of the various response types of the protocol. The networks analyzed here show a better DNS performance than prior research.

The percentage of queries that never receives a response is far lower than the $20+\%$ that [Jung et al., 2002] measured. This study reports an average of a little more than $12 \%$, which can be called acceptable, because of the nonretransmissive nature of the UDP protocol. 
Three quarters of all queries are responded to with a correct IP mapping with on average a latency of $152 \mathrm{~ms} .90 \%$ of these lookups take $275 \mathrm{~ms}$ or less, which is at least twice as fast as earlier research showed.

Clients ask in $9 \%$ of all cases for an IP address for a hostname that does not exist. The amount of queries that are responded to by one of the other return types is very small. Only the amount of format errors is names worthy: a little less than $3.5 \%$. This can be accounted to bugs in client software and transmission errors.

Further research is suggested on DNS data that is not anonymized, so the reasons for the observed format and no such name errors can be analyzed. Location \#4 may look into their network in order to find the reason for the large amount of refused responses.

The strange behavior of the client observed in the traces of location \#1 could be worth to investigate further. Do these actions occur more often and do they bring down the performance of the DNS, or more broad, of the entire network link? Besides this, one might think of changing the settings of the DNS servers in question, so that it responds with a refused message in these situations, which is more logical than a server error response.

\section{ACKNOWLEDGEMENT}

We would like to thank Remco van de Meent for pointing us to and making available the $\mathrm{M} 2 \mathrm{C}$ repository and collecting the data. We also thank Remco van de Meent for his extra information on the DNS system at location \#1. Furthermore, thanks go out to the reviewers for useful comments on the draft version of the paper.

\section{REFERENCES}

ACM SIGCOMM, (2004). Call for papers: Measuring the Internet's Vital Statistics, http://www .acm.org/sigs/sigcomm/ccr/ivs/IVS-CFP.pdf, available: September 5, 2004

Andrews, M. (2004). Negative Caching of DNS Queries (NCACHE), RFC 2308, available at: http://www.rfc-editor.org/rfc.html, November 28, 2004

Brownlee, N., Claffy, K., Nemeth, E., (2001).DNS measurements at a root server, Global Internet 2001, November 2001

Danzig, P.B., Obraczka, K., Kumar, A., (1992).An analysis of wide-area name server traffic: a study of the Internet Domain Name System, ACM SIGCOMM Computer Communication Review, Conference proceedings on Communications architectures \& protocols, Volume 22 Issue 4, October 1992, Pages 281-292

Jung, J., Sit, E., Balakrishnan, H., Robert Morris (2002). DNS Performance and the Effectiveness of Caching, IEEE/ACM Transactions on Networking, Volume 10, No. 5, October 2002, Pages 589-603 
Kurose, J.F., Ross, K.W. (2003). Computer Networking: A Top-Down Approach Featuring the Internet, Addison-Wesley, 2003

Liston, R., Srinivasan, S., Zegura, E., (2002). Diversity in DNS performance measures, Proceedings of the second ACM SIGCOMM Workshop on Internet measurement, November 2002, Pages 19-31

M2C Measurement Data Repository (2004). http://m2c-a.cs.utwente.nl/repository/, updated: unknown, available: September 22, 2004

M2C: Measuring, Modeling and Cost Allocation for Quality of Service, (2004). http://arch.cs.utwente.nl/projects/m2c/, updated: March 2, 2004, available: September 22 , 2004

Mockapetris, P.V. (2004). Domain names - concepts and facilities, RFC 1034, available at: http://www.rfc-editor.org/rfc.html, November 18, 2004

Mockapetris, P.V. (2004). Domain names - implementation and specification, RFC 1035 , available at: http://www.rfc-editor.org/rfc.html, November 18, 2004

Root-servers.org (2004). Root Server Technical Operations Assn, available at: http://www.root-servers.org, November 18, 2004

TCPDUMP public repository (2004). http://www.tcpdump.org/, updated: June 6, 2004

Van de Meent, R. (2003). M2C Measurement Data Repository, University of Twente, Enschede, The Netherlands, December 22, 2003

Van de Meent, R. (2004). University of Twente, email communication during October and November 2004

Wanrooij, W. van (2005). DNS zones revisited, Proceedings of the $11^{\text {th }}$ Open European Summer School, July, 2005, Pages 84-92 